\title{
Photoacoustic imaging of voids in direct wafer bonding
}

\author{
A. C. Gracias \\ Depto. de Fisica, ITA, 12228-901 São José dos Campos, SP, Brazil \\ C. Kuranaga, J. R. Senna, ${ }^{\text {a) }}$ and M. D. Silva \\ LAS, INPE, C.P. 515, 12201-970 São José dos Campos, SP, Brazil
}

(Received 1 February 1999; accepted for publication 5 January 2000)

\begin{abstract}
We demonstrate photoacoustic thermal imaging of the voids occurring at the interface of directly bonded silicon wafers. The wafers are not touched by probes, minimizing wafer contamination, and the technique does not require the outer surfaces of the bonded wafers to be polished. The method is effective for infrared-opaque materials, as demonstrated by coating a silicon bonded pair with bismuth and obtaining an improvement in the contrast. () 2000 American Institute of Physics. [S0034-6748(00)04504-4]
\end{abstract}

\section{INTRODUCTION}

Direct bonding is widely used for joining semiconductor wafers without the use of any intermediate layers. ${ }^{1}$ The basic procedure is to clean the two polished wafer surfaces and bring them into contact, whereupon they bond spontaneously, due both to the van der Waals attraction and to the formation of hydrogen bonds between the OH-terminated surfaces and adsorbed water molecules. During subsequent thermal annealing, water molecules, oxygen, and hydrogen atoms can be eliminated, and covalent bonding across the interface is obtained. ${ }^{2}$

When used to join an oxidized silicon wafer to another silicon wafer, which is later thinned, or from which a thin bonded layer is detached, the method finds one of its most important applications, namely the manufacture of "siliconon-insulator" (SOI) substrates. ${ }^{3}$ The same method has been used to join surfaces of different materials, including metals, semiconductors, and dielectrics, and therefore to obtain substrates which take advantage of the properties of both materials. ${ }^{4}$

Imperfections in the topography of the surfaces, particles, and prebonding surface contamination lead to the presence of voids at the interface before the high-temperature anneal, or to the appearance of voids after the anneal. It is therefore important to monitor the homogeneity of the bonded interface. The standard method of imaging by infrared (IR) transmission is limited by the optical gap of the bonded materials and its applicability decreases as the optical gap of at least one of the materials decreases. Even in the case of silicon to silicon bonding, heavy doping or metal coating of one of the wafers can make this kind of imaging ineffective. Other demonstrated methods of imaging voids at the interface include $x$-ray topography ${ }^{5}$ and acoustic microscopy. ${ }^{6}$ The former detects lattice distortions caused by the voids, and can only be used for voids in bonding of crystalline materials. The latter works for any solid, but requires a liquid coupling medium (typically water) that potentially contaminates the wafer. It is also difficult to apply to

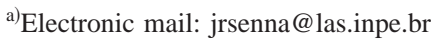

nonannealed bonded pairs, because water can easily penetrate in the interface from the edges of the bonded pair and destroy the weak bond.

Heat conduction, like sound conduction, is not limited by the optical gap, and therefore can be used for imaging both semiconductors and metals. Photoacoustic microscopy has previously been demonstrated for detection of purposely introduced cavities inside solid samples, ${ }^{7}$ and for notches on the back of samples. ${ }^{8}$ In these cases, the defects had thickness of the order of millimeters, while here we demonstrate detection of voids with submicron thickness. The physics and experimental methods of photoacoustic (PA) characterization are well described in the literature, ${ }^{9}$ but we review the basic idea here. The sample surface is illuminated from the front side with intensity-modulated light. The energy absorbed increases the sample local temperature, and heat is transported through the sample by conduction. This heats up the backside of the sample and the fluid (usually air) in the cell facing it, causing a periodic change in pressure which is detected by a microphone.

The imaged region is not touched by any probe, minimizing wafer contamination and risks to the integrity of weak, nonannealed bonds. Other photothermal techniques could be used as well, ${ }^{9}$ which differ in the method used for measuring the increase in the temperature of the back surface. The PA technique has the advantages of requiring contact of this surface to air only and of having no special requirements of polishing of the exterior surfaces. It also has a significant advantage of principle: all the scanning can be done optically, which is a far more robust technique than the mechanical scan used in acoustic microscopy.

The importance of the present method is that the increased absorption of radiation that impairs transmitted light imaging contributes beneficially to PA imaging. This is demonstrated below by imaging a silicon bonded pair whose front surface was coated by a layer of bismuth.

\section{EXPERIMENTAL PROCEDURE}

Pieces of commercial silicon wafers, cut out from oneside polished commercial wafers were cleaned by a standard 


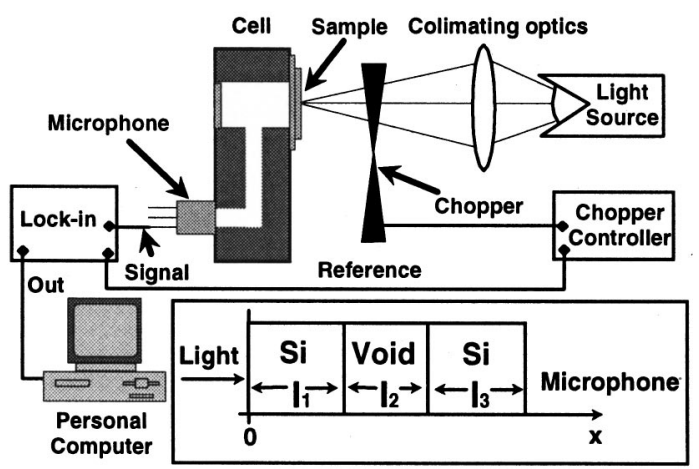

FIG. 1. Scheme of the setup for photoacoustic imaging. The inset shows the sample geometry assumed for the signal calculation.

" $R C A$ " cleaning. ${ }^{10}$ They were then dipped in $\mathrm{HF}: \mathrm{H}_{2} \mathrm{O}(1: 10$ by mass, room temperature) for $20 \mathrm{~s}$, which left them hydrophobic, and rinsed in deionized water for $2 \mathrm{~min}$. This was followed by a hydrophilization treatment. For sample 1 this consisted of immersion in a $\mathrm{NH}_{4} \mathrm{OH}: \mathrm{H}_{2} \mathrm{O}_{2}: \mathrm{H}_{2} \mathrm{O}$ solution (1: 2:18 by volume, $75^{\circ} \mathrm{C}, 10 \mathrm{~min}$ ), rinse in deionized water for $2 \mathrm{~min}$, and spin drying for $2 \mathrm{~min}$ at $3500 \mathrm{rpm}$, followed by contact. The pieces making up sample 2 were treated in an oxygen plasma in a rf reactor for $5 \mathrm{~min}(100 \mathrm{~W}$ rf power, 100 mTorr pressure, $90 \mathrm{sccm}$ oxygen flow) and were contacted as soon as the reactor chamber was open to the atmosphere. All the pairs used in these experiments were found to adhere spontaneously when contacted. They were not annealed at all. Even though samples for imaging were selected exactly because of the presence of voids, as seen by infrared imaging, the adhesion force was sufficient to allow their manipulation.

The samples were imaged by infrared transmitted light, supplied by an incandescent $20 \mathrm{~W}$ light bulb, filtered by a long-pass filter, and the image detected by a near-infrared video camera was captured by a frame grabber. Tests have shown that the interference fringes corresponded to a wavelength distribution centered at $\lambda=1050 \mathrm{~nm}$. The interfacial distance was determined by attributing a distance between the two silicon pieces corresponding to $(2 i-1) \times \lambda / 4$ at the $i$ th dark fringe, with the counting beginning from the region with no fringes.

A small area of each sample was selected for photoacoustic imaging. They were illuminated with white light from a $100 \mathrm{~W}$ tungsten-halogen lamp focused to a small square spot, with a Gaussian-like profile of $480 \mu \mathrm{m}$ full width at half maximum (FWHM), and integrated intensity of $4.7 \mathrm{~mW}$. The incident light was modulated by a chopper at a frequency of $17 \mathrm{~Hz}$. The transmitted heat was detected from the backside, using an air-filled cell at atmospheric pressure into which an electret microphone was inserted. The periodic pressure variation on the microphone was detected synchronously with the light modulation, using a lock-in amplifier. The cell was mounted on a $x-y$ positioner, so that the light spot could be scanned on the sample. The results shown here were obtained with manual scanning, and limited to a $7 \mathrm{~mm}$ by $7 \mathrm{~mm}$ region, with $500 \mu \mathrm{m}$ steps. At each position the amplitude and phase of the microphone voltage were recorded. They were normalized against the signal from a

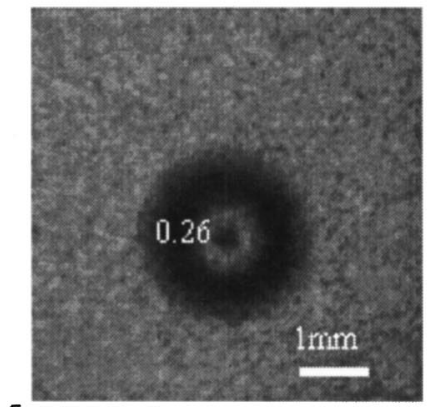

(a)

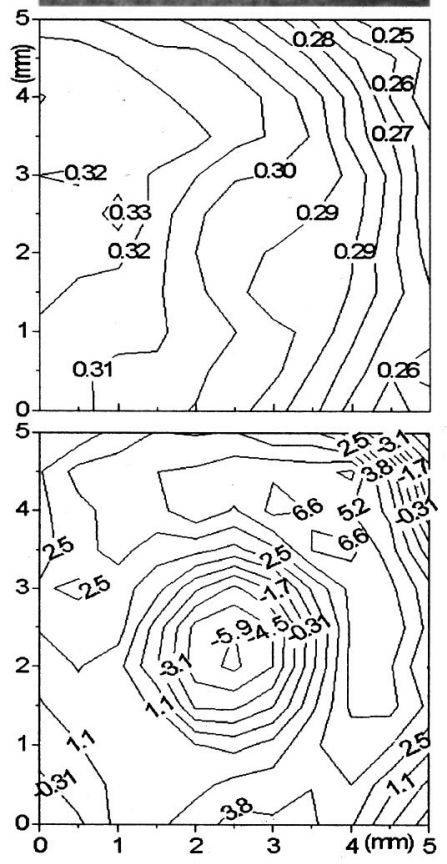

(b)

(c)

FIG. 2. (a) Infrared transmission image through part of sample 1. The spacing in microns, corresponding to a quarter wavelength, is marked on the first fringe. (b) Constant-amplitude curves and (c) constant phase curves, obtained from photoacoustic imaging of the same region.

single wafer sample. The arrangement is shown schematically in Fig. 1.

Figure 2 shows a comparison of infrared transmission imaging and photoacoustic imaging for a region of sample 1, which includes a small round void. Since this void does not reach the boundary of either of the silicon squares in contact, one cannot claim it to be filled with air at ambient pressure. The interface spacing quoted in the figure is nonetheless estimated for a gap filled with material of index of refraction one. Like previous authors, ${ }^{7,11}$ we find that the constantphase contours shown in Fig. 2(c) look more similar to the defect shape in Fig. 2(a) than do the constant-amplitude contours [shown in Fig. 2(b)].

Figure 3 shows a transmitted infrared image of a region of sample 2, in which a large fraction of the area is not bonded. The region where the fringes appear is the nonbonded area. From the spacing of the fringes, the distance between the surfaces is estimated to grow from zero to a maximum of about $1.3 \mu \mathrm{m}$ at the left edge. The lighter, right region is the bonded area, and, according to the prevalent view, ${ }^{2}$ it includes a layer of water molecules between the wafer surfaces. Figures 4(a) and 4(b) are, respectively, the signal constant-intensity and constant-phase maps of the 


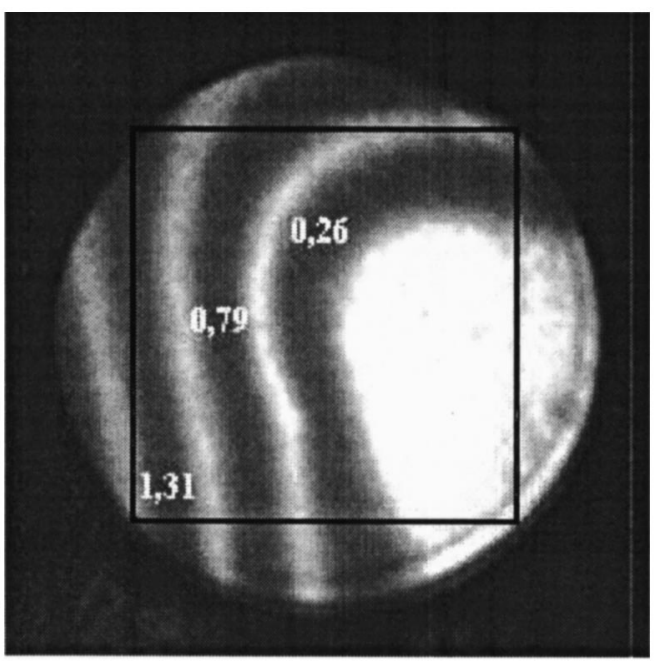

FIG. 3. Infrared transmission image through part of sample 2, attached to the PA cell, showing the circular cell cavity contour and the square scanned region $(7 \mathrm{~mm} \times 7 \mathrm{~mm})$. The spacing in microns, corresponding to an odd number of quarter wavelengths is marked on each fringe.

square region outlined in Fig. 3. This sample was later covered with a $0.1 \mu \mathrm{m}$ thick evaporated layer of bismuth, to make sure the signal obtained was not caused by transmission of the infrared part of the illumination. This resulted in an increase in the signal, due to the better absorption coefficient of the bismuth layer, and we obtained the intensity and phase contours shown in Figs. 4(c) and 4(d).

\section{DISCUSSION}

We have used the thermal diffusion model for the photoacoustic effect, ${ }^{9,12}$ extended to treat a three-layer system. Referring to the inset of Fig. 1, and assuming that heat is generated at $x=0$ at a rate $\operatorname{Re}\left\{l_{0}[\exp (2 \pi i f t)+1]\right\}$ per unit area, we calculated the amplitude $T$ and phase $\phi$ of the periodic temperature at $x=l_{1}+l_{2}+l_{3}$, as a function of $l_{2}$ :

$$
\begin{aligned}
& T\left(l_{2}\right) e^{i \phi\left(l_{2}\right)}=\left(I_{0} / K \sigma\right)\left\{\sinh \left[\sigma\left(l_{1}+l_{3}\right)\right]\right. \\
& \left.\quad+\left(K \sigma l_{2} / K_{2}\right) \sinh \left(\sigma l_{1}\right) \sinh \left(\sigma l_{3}\right)\right\}^{-1}, \\
& \sigma=e^{i \pi / 4} \sqrt{2 \pi f / \alpha},
\end{aligned}
$$

where $K$ and $K_{2}$ are the thermal conductivities of silicon and the intermediate layer, respectively, and $\alpha$ is the thermal diffusivity of silicon. The diffusivity of the intermediate layer does not enter the result as long as its corresponding $\sigma_{2}$ obeys $\sigma_{2} l_{2} \ll 1$ ("thermally thin" layer) and $K_{2} \sigma_{2} \ll K \sigma$. Expression (1) was evaluated for $\alpha=0.814 \mathrm{~cm}^{2} \mathrm{~s}^{-1}, K$ $=1.48 \mathrm{~W} / \mathrm{cm}^{\circ} \mathrm{C}, K_{2}=2.4 \times 10^{-4} \mathrm{~W} / \mathrm{cm}^{\circ} \mathrm{C}$ (appropriate for air), $l_{1}=532 \mu \mathrm{m}$, and $l_{3}=524 \mu \mathrm{m}$. The difference $\phi\left(l_{2}\right)$ $-\phi(0)$ and the ratio $T\left(I_{2}\right) / T(0)$ are plotted in Fig. 5 for $f$ $=17 \mathrm{~Hz}$. The latter ratio is proportional to the signal amplitude ratio. For very thin voids, the phase should be a more sensitive probe of changes in $l_{2}$, with the amplitude sensitivity starting from zero.

The agreement between theory and experiment is reasonable in the case of the bismuth-coated sample. Consider the points in Fig. 4 with vertical coordinate $3.0 \mathrm{~mm}$, and horizontal coordinates $6.8,3.5$, and $0.2 \mathrm{~mm}$. Based on the IR

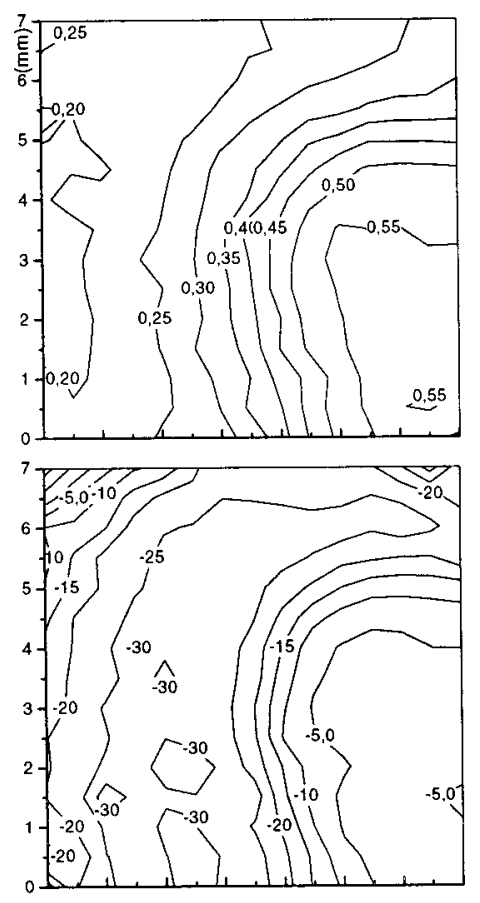

(a)

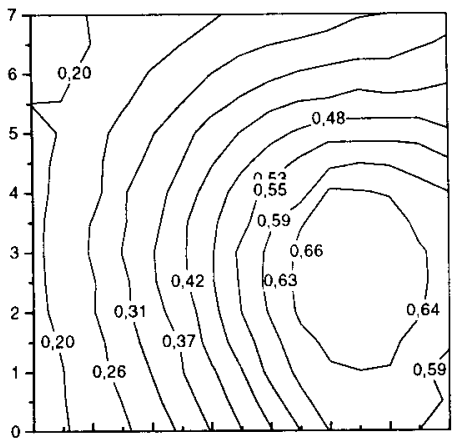

(b)

(c)

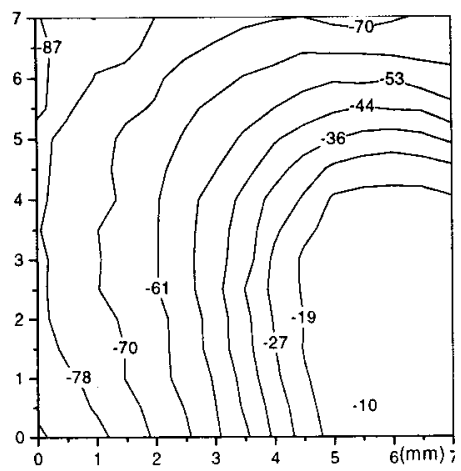

(d)

FIG. 4. (a) Constant-amplitude curves, and (b) constant phase, relative to the rise in the illumination, obtained from photoacoustic imaging of the square region marked in Fig. 3. (c) and (d) same as (a) and (b), respectively, but after coating the sample with bismuth.

fringes in Fig. 3 they correspond to $l_{2}=0,0.26$, and 1.31 $\mu \mathrm{m}$, respectively, and for these $l_{2}$ values the calculation gives relative amplitudes (phases) of $1,0.83$, and $0.33\left(0^{\circ}\right.$, $-27^{\circ}$, and $-64^{\circ}$ ). Figure $4(\mathrm{c})$ gives the amplitude ratio in the sequence 1, 0.86, and 0.31; and Fig. 4(d) gives the phase differences $0^{\circ},-28^{\circ}$, and $-68^{\circ}$.

The simple theoretical model is not appropriate for the case of the uncoated pairs. It assumed light absorption and its complete conversion into heat at the surface. Therefore it does not take into account the effects of nonzero light pen- 


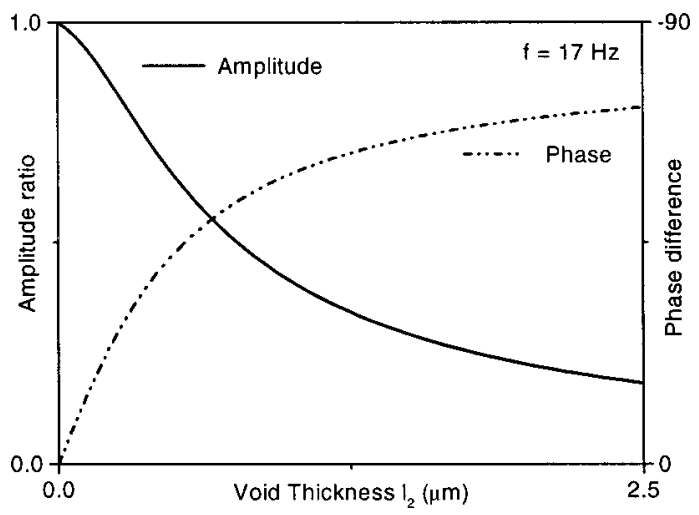

FIG. 5. Calculated amplitude and phase of the temperature at the backside of the sample, normalized by the values at intermediate layer thickness $l_{2}$ $=0$.

etration and of diffusion of excited carriers before they generate heat by nonradiative recombination processes in the bulk. $^{13}$

A simple estimate of the achievable lateral resolution is the thermal diffusion length $s$ given by $|\sigma| s=\sqrt{2}$. Therefore $s$ is proportional to the inverse square root of the frequency. For silicon, and with $f=17 \mathrm{~Hz}$, this gives $s=1.2 \mathrm{~mm}$, and a resolution of $0.1 \mathrm{~mm}$ would require a chopping frequency of $2600 \mathrm{~Hz}$. With a pixel side given by the lateral resolution, and a minimum time of $f^{-1}$ to measure the signal from a pixel, one estimates the imaging throughput at maximum resolution to be of the order of $\alpha / \pi$, corresponding to 0.26 $\mathrm{cm}^{2} / \mathrm{s}$ in silicon.

In conclusion, we demonstrated photoacoustic imaging of voids in direct bonded silicon. We obtained a remarkably high contrast between the well-contacted areas and the areas with thin voids buried between half-millimeter thick wafers. By coating the silicon bonded pairs with bismuth and obtain- ing an improvement in the contrast, we demonstrated the effectiveness of the method for infrared-opaque materials and its feasibility for monitoring bonding to metals and narrow-gap semiconductors. We consider this a promising technique for situations where other available techniques are inappropriate (noncrystalline, opaque materials, and nonannealed bonds).

\section{AKNOWLEDGMENTS}

We thank Dr. I. A. Maia for help in setting up and operating the plasma reactor. This work was partially funded by Fapesp 95/1493-0, A. C. G. is supported by Fapesp fellowship 97/03862-9, and C. K. by CNPq fellowship 381780/ 97-0.

${ }^{1}$ I. M. Shimbo, K. Furukawa, K. Fukuda, and K. Tanzawa, J. Appl. Phys. 60, 2987 (1986).

${ }^{2}$ R. Stengl, T. Tan, and U. Gosele, Jpn. J. Appl. Phys., Part 1 28, 10 (1989); for a review, see Q. Y. Tong and U. Gosele, Semiconductor Wafer Bonding: Science and Technology (Wiley, New York, 1998).

${ }^{3}$ J. B. Lasky, Appl. Phys. Lett. 48, 78 (1986).

${ }^{4}$ G. A. C. M. Spierings and J. Haisma, J. Electrochem. Soc. 92, 18 (1991); W. P. Maszara, J. Electrochem. Soc. 138, 341 (1991).

${ }^{5}$ J. Haisma, G. A. C. Spierings, U. K. P. Biermann, and J. A. Pals, Jpn. J. Appl. Phys., Part 1 28, 1426 (1989).

${ }^{6}$ R. D. Black, S. D. Arthur, R. S. Gilmore, N. Lewis, E. L. Hall, and R. D. Lillquist, J. Appl. Phys. 63, 2773 (1988).

${ }^{7}$ G. Busse, Appl. Phys. Lett. 35, 759 (1979).

${ }^{8}$ H. Endoh, Y. Hiwatashi, and T. Hoshimiya, Jpn. J. Appl. Phys., Part 1 36, 3312 (1997)

${ }^{9}$ A. Rosencwaig, Photoacoustics and Photoacoustic Spectroscopy (Wiley, New York, 1980); H. Vargas and L. C. M. Miranda, Phys. Rep. 161, 43 (1988).

${ }^{10}$ W. Kern and D. A. Puotinen, RCA Rev. 31, 187 (1970).

${ }^{11}$ A. Rosencwaig and G. Busse, Appl. Phys. Lett. 36, 725 (1980).

${ }^{12}$ A. Rosencwaig and A. Gersho, J. Appl. Phys. 47, 64 (1976).

${ }^{13}$ A. Pinto Neto, H. Vargas, N. F. Leite, and L. C. M. Miranda, Phys. Rev. B 41, 9971 (1990). 